\title{
Diseño y validación de un ensayo de minisecuenciación múltiple para detectar polimorfismos asociados con Sindrome Metabólico
}

\author{
Design and validation of a multiple minisequencing assay to \\ detect polymorphisms associated with Metabolic Syndrome
}

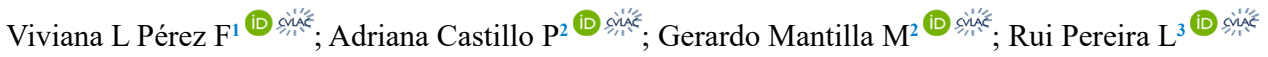

Forma de citar: Pérez V, Castillo A, Mantilla G, Pereira R. Diseño y validación de un ensayo de minisecuenciación múltiple para detectar polimorfismos asociados con síndrome metabólico. Salud UIS. 2021; e21031. doi:

https://doi.org/10.18273/saluduis.53.e:21031 (c) (1)

\section{Resumen}

Introducción: es importante identificar los polimorfismos de interés clínico en patologías complejas como el Síndrome Metabólico. Por esto, las metodologías para su evaluación deben estar diseñadas y validadas correctamente, esto permite optimizar recursos y tiempo en la genotipificación y detección correcta de los alelos presentes en los individuos. Objetivo: diseñar y validar una PCR múltiple, seguida de detección por minisecuenciación, para la genotipificación de ocho polimorfismos de nucleótido simple ubicados en el gen del Receptor Beta 3-Adrenérgico (rs4994 y rs4998), gen de la Apolipoproteina A5 (rs3135506 y rs2075291), gen de la Adiponectina (rs1501299 y rs2241766) y gen del Receptor Activador de la Proliferación de los Peroxisomas tipo gamma (rs1801282 y rs 1800571), asociados con el síndrome metabólico. Materiales y métodos: se diseñaron 24 cebadores para la amplificación y detección de ocho polimorfismos de nucleótido sencillo ubicados en cuatro genes candidatos a estar asociados con el síndrome metabólico, usando el software Primer $3^{\circledR}$. Dieciséis fueron diseñados para amplificar los polimorfismos y ocho para detectarlos por minisecuenciación. Las estructuras secundarias entre los cebadores se verificaron con el software Autodimer. Los polimorfismos se amplificaron simultáneamente y los fragmentos amplificados se acoplaron a las sondas diseñadas para detectar por minisecuenciación el alelo presente, por medio de bases marcadas con fluorocromos. Finalmente, los alelos fueron detectados por electroforesis capilar en un analizador genético ABI 310 y se interpretaron con el software GeneMapper ${ }^{\circledR}$. La validación del multiplex se realizó genotipando 20 muestras de individuos, cada uno de ellos autorizó este procedimiento por medio del consentimiento informado. Resultados: se obtuvieron los perfiles genéticos de los 20 controles genotipados, a partir de la amplificación múltiple, seguida de minisecuenciación, diseñada y validada para detectar los ocho polimorfismos. Conclusión: se diseñó y validó un ensayo para la detección simultánea de los polimorfismos, ubicados en cuatro genes asociados con el Síndrome metabólico. Los cuales pueden ser empleados como referencia para futuros estudios poblacionales.

Palabras clave: APOA5; PPARG; Adiponectina; ADBR; Síndrome Metabólico; Polimorfismo de Nucleótido Sencillo (SNP); SNaPshot ${ }^{\mathbb{R}}$; Electroforesis Capilar (EC).

1. Universidad de Santander. Bucaramanga, Colombia.

2. Universidad Industrial de Santander. Bucaramanga, Colombia.

3. University of Porto. Oporto, Portugal.

Correspondencia: Viviana Lucia Pérez Forero. Dirección: Calle 70 55-210, Bucaramanga, Colombia. Teléfono: +57 (7) 6516500 . Correo electrónico: viviana.perez@udes.edu.co 


\begin{abstract}
Introduction: It is important to identify the polymorphisms of clinical interest in complex pathologies such as Metabolic Syndrome. Therefore, the methodologies for its evaluation must be designed and validated correctly, this permits optimization of resources and time in genotyping and correct detection of the alleles present in individuals. Objective: To design and validate a multiplex PCR, followed by detection by minisequencing, for the genotyping of eight single nucleotide polymorphisms located in the Beta 3-Adrenergic Receptor gene (rs4994 and rs4998), Apolipoprotein A5 gene ( $r s 3135506$ and rs2075291), Adiponectin gene ( $r s 1501299$ and rs2241766) and gammatype Peroxisome Proliferation Activating Receptor gene ( $r$ s1801282 and $r s 1800571)$, associated with metabolic syndrome. Materials and methods: Twenty-four primers were designed for the amplification and detection of eight single nucleotide polymorphisms located in four candidate genes to be associated with the metabolic syndrome, using the Primer $3^{\circledR}$ software. Sixteen were designed to amplify the polymorphisms and eight to detect them by minisequencing. The secondary structures between the primers were verified with Autodimer software. The polymorphisms were simultaneously amplified, and the amplified fragments were coupled to probes designed to minisequence the present allele using fluorochrome-labeled bases. Finally, the alleles were detected by capillary electrophoresis using an ABI 310 genetic analyzer and analyzed with the GeneMapper ${ }^{\circledR}$ software. The validation of the multiplex was performed by genotyping 20 individual samples, each of them authorized this procedure through informed consent. Results: The genetic profiles of the 20 genotyped controls were obtained, from multiple amplification, followed by minisequencing, designed and validated to detect the eight polymorphisms. Conclusion: An essay was designed and validated for the simultaneous detection of polymorphisms, located in four genes associated with metabolic syndrome, and can used as a reference for future population studies.
\end{abstract}

Keywords: APOA5; PPARG; Adiponectin; ADBR; Metabolic Syndrome; Single nucleotide Polymorphism (SNP), $\mathrm{SNaPshot}^{\circledR}$; Capillary electrophoresis (CE).

\section{Introducción}

Los polimorfismos de nucleótido sencillo (SNP) son sustituciones de una única base en posiciones específicas dentro del Acido Desoxirribonucleico (ADN). Su valoración en estudios genéticos es muy usada debido a que son los polimorfismos más abundantes en el genoma humano, representan alrededor del $85 \%$ de la variación genética humana, tienen una baja tasa de mutación, (del orden de 10 $0^{-8}$ ) por tanto son muy estables, y pueden analizarse en amplicones producto de la Reacción en Cadena de la Polimerasa (PCR) de tamaño pequeño, entre otros atributos ${ }^{1}$. Para su detección se usan diversas metodologías como la secuenciación directa, hibridización alélica específica (ASO), los microarreglos, las sondas de Hibridación TaqMan, la espectrometría de masas (MALDI-TOF), la secuenciación masiva en paralelo (MPS) y la minisecuenciación por $\mathrm{SNaPshot}{ }^{\mathbb{R 2}, 3}$.

La caracterización de un conjunto de SNP puede emplearse en estudios de asociación y se relacionan con el riesgo de desarrollar alguna patología; la detección de la base polimórfica es importante para evaluar el componente genético de las enfermedades complejas. Los estudios funcionales basados en polimorfismos son eficientes porque se centran en los SNP con mayor probabilidad de estar asociados y permiten a su vez identificar tanto alelos comunes como raros en regiones funcionales ${ }^{4}$.

Esta valoración es útil en entidades multifactoriales como el Síndrome Metabólico (SM) el cual es una agrupación de hallazgos clínicos como hipertensión, obesidad, dislipidemia e hiperglicemia o insulino resistencia, en la que convergen diversos factores ambientales, el estilo de vida y un componente genético $^{5-7}$. Según la Organización Mundial de la Salud (OMS), la prevalencia mundial de obesidad se ha duplicado en las últimas tres décadas y, al menos un tercio de los adultos mayores de 20 años tienen sobrepeso u obesidad.

Con el aumento de la obesidad, se espera que la prevalencia del SM también aumentará en paralelo, dado que casi la mitad de la carga de diabetes y una cuarta parte de la carga de enfermedad cardíaca es atribuible al sobrepeso u obesidad ${ }^{5}$.

Hasta el momento se han identificado más de 200 polimorfismos de tipo SNP en cerca de 100 genes, que han sido relacionados en diversas investigaciones con los criterios diagnósticos de SM. Entre los genes propuestos como candidatos a contribuir al 
desarrollo de SM están los genes del Receptor Beta 3-Adrenérgico (ADBR3), la Apolipoproteína A5 (APOA5), la Adiponectina (ADIPOQ) y del receptor activador de la proliferación de los peroxisomas tipo gamma (PPARG) $)^{8-10}$.

El gen ADBR3 localizado en el cromosoma 8, juega un papel importante en la regulación de la termogénesis y la lipolisis en tejido adiposo blanco y es un gen candidato en sujetos obesos, debido a que mutaciones funcionales alteran la lipólisis estimulada por catecolaminas causando una disminución en la tasa metabólica basal ${ }^{11}$. El gen APOA5 se localiza en el cromosoma 11, codifica para una proteína involucrada en el metabolismo de los triacilgliceroles y la glucosa, y ha sido asociado a hipertrigliceridemia y riesgo de desarrollar $\mathrm{SM}^{12}$. El gen ADIPOQ localizado en el cromosoma 3, codifica para una proteína específica del tejido adiposo que circula en el plasma, cuyos niveles están inversamente correlacionados con la resistencia a la insulina y expresión reducida en presencia de obesidad ${ }^{13,14}$. El gen PPARG localizado en el cromosoma 3, codifica para receptores nucleares encargados de regular la expresión de diversos genes implicados en el metabolismo de los lípidos y la glucosa; su expresión ha sido comprobada en diversos tejidos como el adiposo donde juegan un papel crucial en la diferenciación celular y la regulación en la expresión de otros genes involucrados en el metabolismo energético ${ }^{15,16}$.

En estos genes se han estudiado diferentes SNPs para establecer su asociación con el SM, en este estudio se seleccionaron dos polimorfismos de cada uno de estos genes, de acuerdo con los criterios clínicos de la base de datos clinvar, así: del gen ADBR3 el rs4994 (Trp64Arg) y $r s 4998(250 \mathrm{G}>\mathrm{C})$, del gen APOA5 el $r s 3135506$ (Ser19Trp) y $r s 2075291$ (Gly185Cys), del gen ADIPOQ el rs1501299 (SNP276) y rs2241766 (Gly15=) y del gen PPARG el rs 1801282 (Pro12Ala) y rs1800571 (Pro85Gln) ${ }^{8,12,17-19}$.

Dado que no se encontró reportado en la literatura ningún diseño metodológico para detectar simultáneamente estos polimorfismos de interés clínico, este trabajo presenta el diseño y validación de cebadores, así como el desarrollo de una PCR múltiple, seguida de minisecuenciación con la metodología $\mathrm{SNaPshot}{ }^{\circledR}$ y detección por electroforesis capilar, para su identificación. Se seleccionó la minisecuenciación con $\mathrm{SNaPshot}{ }^{\circledR}$ porque es una técnica robusta, posee mayor sensibilidad que la secuenciación estándar, es de bajo costo, rápida, requiere poca cantidad de
$\mathrm{ADN}$, permite la detección de variantes menores y pueden ser empleados amplicones de PCR pequeños. Adicionalmente permite identificar hasta 29 SNP de diferentes loci en una sola reacción y la literatura científica muestra su utilidad en la detección y validación de $\mathrm{SNP}^{20}$.

\section{Metodología}

La detección de los SNPs se realizó mediante la minisecuenciación que se basa en la alta precisión del ADN polimerasa para la incorporación del nucleótido de interés. Los segmentos de ADN que contenían los SNPs a evaluar se amplificaron en una PCR múltiple y posteriormente cada fragmento amplificado fue hibridado con un cebador no marcado (sonda) específico para cada SNP y diseñado para unirse al ADN diana de tal forma que su extremo 3' finalizaba en el nucleótido inmediatamente adyacente al SNP. Posteriormente una ADN polimerasa extendió la cadena incorporando un único dideoxinucleótido trifosfato (ddNTP) marcado con un fluorocromo específico para cada base nitrogenada, usando el kit SNaPshot ${ }^{\circledR}$ (Applied Biosystems). El ddNTP se unió en la posición del SNP, o en la complementaria de acuerdo con el diseño de la sonda. Los productos se separaron por electroforesis capilar en un secuenciador de ADN automatizado ${ }^{2}$.

El diseño y desarrollo del ensayo para la detección múltiple de los ocho SNPs asociados con el SM, se desarrolló así:

\section{Ubicación de los SNPs}

Se identificaron los genes y SNPs de interés clínico relacionados con el SM en la base de datos dbSNP y ClinVar disponibles en NCBI. Como criterios de inclusión se contemplaron: la ubicación del SNP en una región codificante, que generará una mutación no sinónima, estar validado teniendo en cuenta que el alelo de menor frecuencia fuese mayor de 0,001 y haber sido secuenciado en el proyecto de los Mil Genomas (https://www.internationalgenome.org/). La ubicación y variación en la secuencia de los ocho SNPs se presenta en las tablas 1 y 2 . Se revisaron todos los SNPs ubicados dentro de los cebadores diseñados, para elegir solo los que reportaban una frecuencia alélica del menor alelo (MAF) mayor de 0,001.

\section{Diseño de Cebadores}

Los cebadores para la amplificación por PCR, y para la minisecuenciación fueron diseñados para tener una temperatura de hibridación alrededor de 
$60{ }^{\circ} \mathrm{C}$ y una longitud del producto de 80 a $350 \mathrm{pb}$ usando el software Primer 3 (https://primer3. ut.ee/). Se seleccionaron secuencias de cebadores de minisecuenciación corriente arriba y corriente abajo adyacente al sitio SNP, con una longitud y temperatura de fusión (Tm) apropiadas ${ }^{21,22}$.

Las secuencias de la base de datos del National Centre for Biotechnology Information fueron comparadas utilizando la herramienta de búsqueda de alineación de nucleotidos Basic Local Alignment Search Tool (BLAST), para probar los cebadores (de PCR multiplex y SNaPhot) contra posibles secuencias repetitivas y homologías de secuencia. Para evitar posibles estructuras en horquilla y las interacciones cebador-cebador en la PCR multiplex, estos se probaron utilizando el software AutoDimer (https://strbase.nist.gov/ AutoDimerHomepage/AutoDimerProgramHomepage. htm). Además, se revisaron las posibles variantes de las bases nitrogenadas y la frecuencia descrita dentro de la zona de los primers diseñados, para reemplazarlas siguiendo la nomenclatura IUPAC (https://www. bioinformatics.org/sms/iupac.html), ampliando así el éxito de la amplificación, si estaba presente.
El diseño de los cebadores de $\mathrm{SNaPshot}^{\circledR}$ para la detección de varios SNPs fue una parte fundamental en el desarrollo de la técnica, los cebadores debían permitir que el ddNTP marcado se incorporara exactamente en la posición correspondiente al polimorfismo, no debía existir hibridación cruzada entre los cebadores y debían poderse diferenciar en una electroforesis. Para diferenciarlos se les incorporó una cola de nucleótidos con secuencias no homólogas de polinucleotidos (dTAGGTGCCACGTCGTGAAAGTCTGACAA) de distinto tamaño en el extremo $5^{\prime}$ de cada cebador logrando que los productos finales de la minisecuenciación tuvieran diferencias en el tamaño del fragmento evaluado de al menos cuatro nucleótidos de longitud y evitar así la superposición de los productos ${ }^{23,24}$.

Seguidamente se confirmaron las temperaturas de anillamiento y el tamaño de los segmentos amplificados usando UCSC In-Silico PCR con una concentración de sales de $50 \mathrm{mM}$ y de cebadores de $50 \mathrm{nM}$. Las secuencias de los cebadores para la amplificación por PCR y para la reacción de minisecuenciación se muestran en las Tablas 1 y 2 , respectivamente.

Tabla 1. Secuencias de los cebadores diseñados para la amplificación por PCR.

\begin{tabular}{|c|c|c|c|c|c|c|}
\hline SNPs & Cebadores & $\begin{array}{c}\text { Tamaño } \\
\text { (pb) }\end{array}$ & $\begin{array}{l}\text { TM } \\
{ }^{\circ} \mathbf{C}^{\dagger}\end{array}$ & $\% \mathbf{G C}^{\ddagger}$ & $\begin{array}{c}\text { Tamaño } \\
\text { Amplificado } \\
\text { (pb) }\end{array}$ & $\begin{array}{l}\text { Posición chr } \\
\text { (grch37.p5) }\end{array}$ \\
\hline \multirow{2}{*}{$\begin{array}{l}\text { Trp64Arg } \\
\text { (rs4994) }\end{array}$} & 5'-CСТTCCTTCYTTCCCTACCG-3'(F) & 20 & 60,1 & 55,0 & \multirow[t]{2}{*}{263} & \multirow[t]{2}{*}{ chr8:37966280 } \\
\hline & 5'-AACACGTTGGTCATGGTCTG-3'(R) & 20 & 59,4 & 50,0 & & \\
\hline \multirow{2}{*}{$\begin{array}{l}250 G>C \\
(r s 4998)\end{array}$} & 5'-CCAAGTGGGTTTTYACCATC3'(F) & 20 & 60,2 & 50,0 & \multirow{2}{*}{185} & \multirow{2}{*}{ chr8:37963968 } \\
\hline & 5'-AGYTCTCTTTGCCCTAAGCA-3'(R) & 20 & 59,4 & 50,0 & & \\
\hline \multirow{2}{*}{$\begin{array}{l}\text { Ser19Trp } \\
\text { (rs3135506) }\end{array}$} & 5'-CCG CTG TCT CCT CCC TTC-3'(F) & 19 & 59,9 & 63,1 & \multirow{2}{*}{268} & \multirow{2}{*}{ chr11:116791691 } \\
\hline & 5'-TCTCCGACCCTGACTTCAAC-3'(R) & 20 & 60,2 & 55,0 & & \\
\hline \multirow{2}{*}{$\begin{array}{l}\text { Gly185Cys } \\
\text { (rs2075291) }\end{array}$} & 5'-AMTGAAGCCCTACACGATGG-3'(F) & 20 & 60,1 & 55,0 & \multirow{2}{*}{213} & \multirow{2}{*}{ chr11:116790676 } \\
\hline & 5'-ACCAGGCTCTCGGCGTAT-3'(R) & 18 & 60,8 & 61,1 & & \\
\hline \multirow{2}{*}{$\begin{array}{l}\text { SNP276 } \\
\text { (rs1501299) }\end{array}$} & 5'-AGATGCAGCAAAGCCAAAGT-3'(F) & 20 & 60,0 & 45,5 & \multirow{2}{*}{196} & \multirow{2}{*}{ chr3:186853334 } \\
\hline & 5'-GGCCTCTTTCATCACAGACC-3'(R) & 20 & 59,7 & 55,0 & & \\
\hline \multirow{2}{*}{$\begin{array}{l}\text { Gly15= } \\
\text { (rs2241766) }\end{array}$} & 5'-TGTGTGTGTGGGGTCTGTCT-3'(F) & 20 & 60,0 & 55,0 & \multirow{2}{*}{130} & \multirow{2}{*}{ chr3:186853103 } \\
\hline & 5'-TTGARTCGTGGTTTCCTGGT-3'(R) & 20 & 60,5 & 50,0 & & \\
\hline \multirow{2}{*}{$\begin{array}{l}\text { Pro12Ala } \\
\text { (rs1801282) }\end{array}$} & 5'-TCCATGCTGTTATGGGTGAA-3'(F) & 20 & 59,9 & 45,0 & \multirow{2}{*}{186} & \multirow{2}{*}{$\operatorname{chr} 3: 12351626$} \\
\hline & 5'-CAAACACAACCTGGAAGACAAA-3'(R) & 22 & 60,0 & 40,9 & & \\
\hline \multirow{2}{*}{$\begin{array}{l}\text { Pro85Gln } \\
\text { (rs1800571) }\end{array}$} & 5'-ATCAAAGTGGAGCCTGCATC-3'(F) & 20 & 60,2 & 50,0 & \multirow{2}{*}{104} & \multirow{2}{*}{ chr3:12381349 } \\
\hline & 5'-CGACATTCAATTGCCATGAG-3'(R) & 20 & 60,1 & 45,0 & & \\
\hline
\end{tabular}

$\dagger$ Temperatura de anillamiento, $\ddagger$ Porcentaje de Guanina y Citosina 
Tabla 2. Secuencias de cebadores tipo sonda empleados para la técnica de SNaPshot.

\begin{tabular}{|c|c|c|c|}
\hline Gen & Secuencia de sondas & Tamaño (pb) & Base identificada \\
\hline \multirow{2}{*}{ ADBR3 } & 5'-ATCGTGGCCATCGCC-3'(F) & 15 & $\mathrm{~T} / \mathrm{C}$ \\
\hline & 5'-cgtcgtgaaagtctgacaaCCTCAGTGGTAGTGTCCAG-3'(F) & 38 & $\mathrm{C} / \mathrm{G}$ \\
\hline \multirow{2}{*}{ APOA5 } & 5'-tgacaaCCTCTCCACAGCGTTTT-3'(R) & 23 & $\mathrm{C} / \mathrm{G}$ \\
\hline & 5'-actaggtgccacgtcgtgaaagtctgAGCTCTTTGAAGCGGY-3'(R) & 42 & $\mathrm{G} / \mathrm{T}$ \\
\hline \multirow{2}{*}{ ADIPOQ } & 5'-acaаTCTAGGCCTTAGTTAATAATGAATG-3’(F) & 29 & $\mathrm{~A} / \mathrm{C}$ \\
\hline & 5'- ctctctctctctctctgaaagtctgacaaGTGGTTTCCTGGTCATG-3'(R) & 46 & $\mathrm{G} / \mathrm{T}$ \\
\hline \multirow{2}{*}{ PPARG } & 5'-gtgaaagtctgacaaGTGAAGGAATCGCTTTCTG-3'(R) & 34 & $\mathrm{C} / \mathrm{G}$ \\
\hline & 5' aaactaggtgccacgtcgtgaaagtctgacaaAAGTGGAGCCTGCATCTC-'(F) & 50 & $\mathrm{C} / \mathrm{A}$ \\
\hline
\end{tabular}

\section{Muestras}

Se recolectaron muestras de sangre periférica de 20 donantes no emparentados previo consentimiento informado. El ADN genómico fue aislado, usando el kit comercial PrepFiler ${ }^{\mathrm{TM}}{ }^{\circledR}$ Forensic DNA ${ }^{\circledR}$ (Applied Biosytems).

\section{PCR múltiple}

Se diseñó una PCR múltiple para amplificar los ocho SNPs seleccionados, en una sola reacción de amplificación con volumen final de 25 uL así: 12,5 uL de $2 \mathrm{X}$ de PCR Multiplex QIAGEN $^{\circledR}$, los primer en concentración final $0,2 \mathrm{uM}, 3 \mathrm{uL}$ de $\mathrm{H}_{2} \mathrm{O}$ y $20 \mathrm{ng}$ de ADN genómico. Las condiciones de termociclado fueron $95^{\circ} \mathrm{C}$ por $15 \mathrm{~min}$, luego 30 ciclos de $94{ }^{\circ} \mathrm{C}$ por $30 \mathrm{~s}, 60{ }^{\circ} \mathrm{C}$ por $90 \mathrm{~s}, 72{ }^{\circ} \mathrm{C}$ por $60 \mathrm{~s}$, y una extensión final a $72{ }^{\circ} \mathrm{C}$ por $60 \mathrm{~min}$.

\section{Purificación del amplificado}

El exceso de cebadores y dNTPs del amplificado fue eliminado mezclando 2,5 uL del producto amplificado con 1 uL de ExoSAP-IT (Amersham Biotech ${ }^{\circledR}$ ). Se llevó a incubación a $37^{\circ} \mathrm{C}$ por 45 min, seguido de incubación a $85^{\circ} \mathrm{C}$ por $15 \mathrm{~min}$, para inactivar la enzima.

\section{Minisecuenciación múltiple}

Se realizó una segunda secuenciación con un volumen final de $6 \mathrm{uL}$, incluyendo $2,5 \mathrm{uL}$ de reacción de $\mathrm{SNaPshot}^{\circledR}$ (Applied Biosystems), 2 uL del producto de PCR y 1,5 uL de la mezcla de cebadores de extensión diseñados (las concentraciones finales estuvieron entre $0,15$ y $0,6 \mathrm{umol} / \mathrm{L})$. Las condiciones de termociclador fueron: 30 ciclos de $96{ }^{\circ} \mathrm{C}$ por $10 \mathrm{~s}, 55^{\circ} \mathrm{C}$ por $5 \mathrm{~s} \mathrm{y}$ $60{ }^{\circ} \mathrm{C}$ por $30 \mathrm{~s}$.

\section{Purificación de los productos de SNaPshot ${ }^{\circledR}$}

Para remover los ddNTPs no incorporados, se mezclaron $6 \mathrm{uL}$ del producto de minisecuenciación, con 1 unidad de fosfatasa alcalina de camarón (SAP, Amersham Biotech $^{\circledR}$ ). Se incubó a $37^{\circ} \mathrm{C}$ por 80 min, seguido de $80{ }^{\circ} \mathrm{C}$ por 15 min para inactivar la enzima.

\section{Detección de SNPs por electroforesis capilar}

Se mezclaron $0,5 \mathrm{uL}$ del producto de la minisecuenciación purificado con $12 \mathrm{uL}$ de formamida Hi-DiTM y $0,3 \mathrm{uL}$ de marcador de peso GeneScan-120 LIZ ${ }^{\circledR}$ (Applied Biosystems), posteriormente se denaturaron las muestras a $95^{\circ} \mathrm{C}$ por $5 \mathrm{~min}$. Las electroforesis fueron corridas en un analizador genético ABI 310 usando polímero POP-4 para visualizar cada uno de los ddNTPs incorporados. Los datos se analizaron con el software GeneMapper ${ }^{\circledR}$ ID v3.2 (Applied Biosystems) para asignar los alelos y determinar el perfil genético de la muestra ${ }^{25}$.

\section{Validación del método diseñado}

La validación del diseño de los primers y de la PCR múltiplex, se realizó siguiendo los criterios de la norma ISO-NTC/IEC 17025 de 2017 para validación de métodos ${ }^{26}$. Las cuales se mencionan a continuación:

\section{Umbral analítico}

Se calculó como el valor que, en las condiciones analíticas empleadas por el laboratorio, permitió establecer la altura de un pico a partir de la cual el pico observado, era en realidad un producto de PCR. Los valores de la señal de fluorescencia se tomaron las unidades de fluorescencia relativa (RFU) de cada pico $^{24,27}$. 


\section{Especificidad}

Fue evaluada como la capacidad del método para detectar la ubicación y secuencia del gen de interés en el genoma humano. Se determinó mediante el análisis de los resultados obtenidos por secuenciación directa y por minisecuenciación.

\section{Exactitud}

Se determinó como la capacidad del método de PCR múltiple y del método $\mathrm{SNaPshot}^{\circledR}$ para detectar inequívocamente cada uno de los SNPs de interés, comparando los resultados obtenidos por minisecuenciación y por secuenciación directa.

\section{Precisión}

Se evaluó mediante la repetibilidad obtenida en cinco muestras, procesadas tres veces por un mismo funcionario; y mediante reproducibilidad, valorada a partir de la evaluación de los resultados obtenidos en las mismas cinco muestras procesadas a ciegas, por otro funcionario, utilizando otro termociclador del laboratorio ${ }^{28}$.

\section{Resultados}

\section{Detección de los SNPs}

Con los cebadores diseñados se secuenciaron 20 muestras controles para los polimorfismos: rs4994 (Trp64Arg) y rs4998 (250G $>$ C) del gen ADBR3, $r s 3135506$ (Ser19Trp) y rs2075291 (Gly185Cys) del gen APOA5, rs1501299 (SNP276) y rs2241766 (Gly15=) del gen ADIPOQ y rs 1801282 (Pro12Ala) y rs 1800571 (Pro85Gln) del gen PPARG. Las secuencias obtenidas fueron interrogadas en BLAST y en todos los casos se pudo confirmar que los fragmentos amplificados correspondían a la secuencia esperada de cada gen, lo cual puede ser verificado con la Figura 1 donde se observa el resultado de la secuenciación de una muestra para el polimorfismo ApoA5.

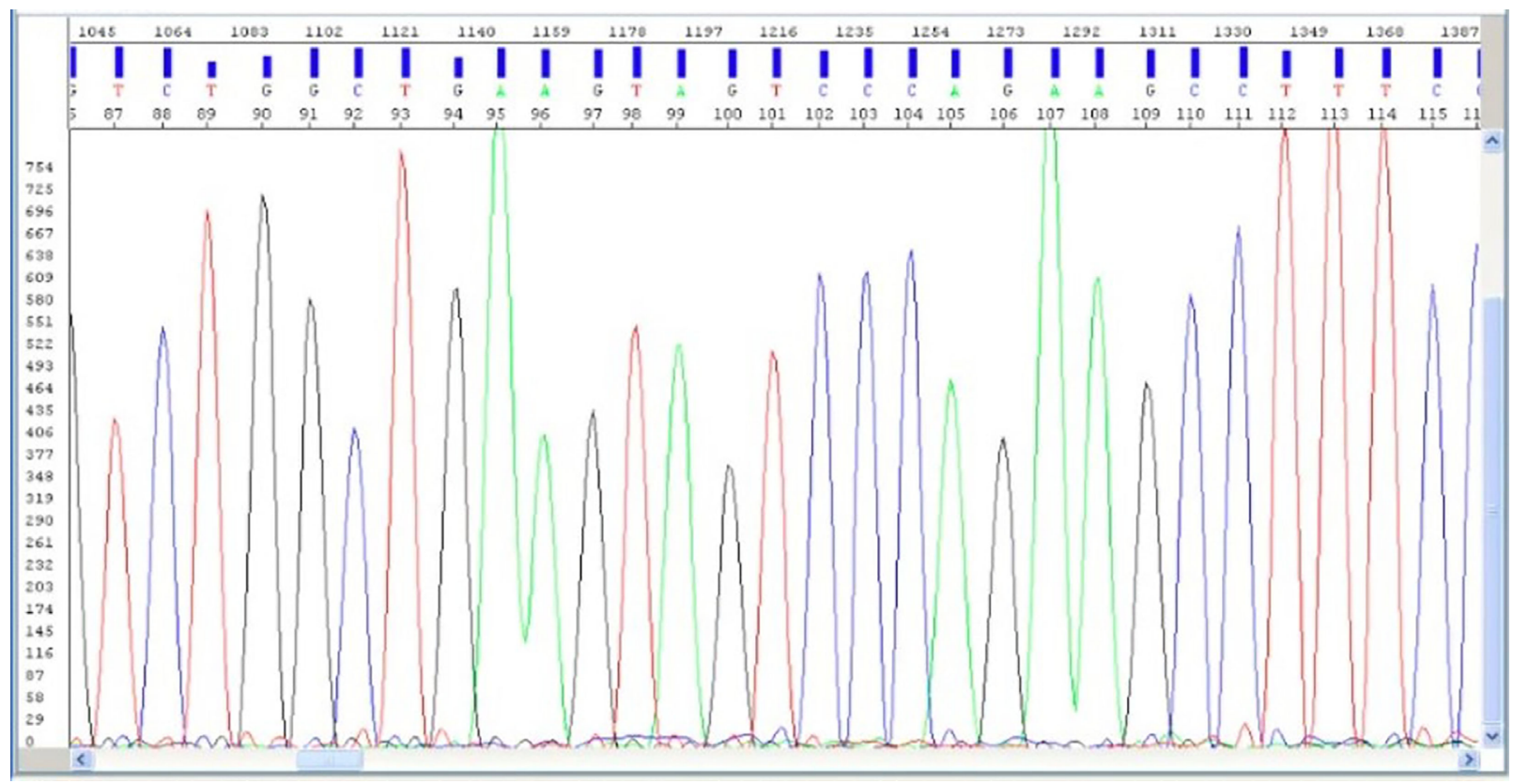

Figura 1. Resultado de la secuenciación directa de un segmento del gen APOA.

A continuación, se realizó el montaje de la PCR múltiple con los cebadores diseñados. La amplificación se verificó en un gel de agarosa $\mathrm{Biomol}^{\circledR}(100 \mathrm{~V}$ por $30 \mathrm{~min})$ al $2 \%$. Posteriormente se realizó la minisecuenciación por $\mathrm{SNaPshot}{ }^{\circledR}$, usando las sondas específicas diseñadas para la detección de cada polimorfismo. En todos los 20 controles evaluados se obtuvieron resultados para los ocho SNPs investigados. Los resultados se muestran en la Figura 2. 


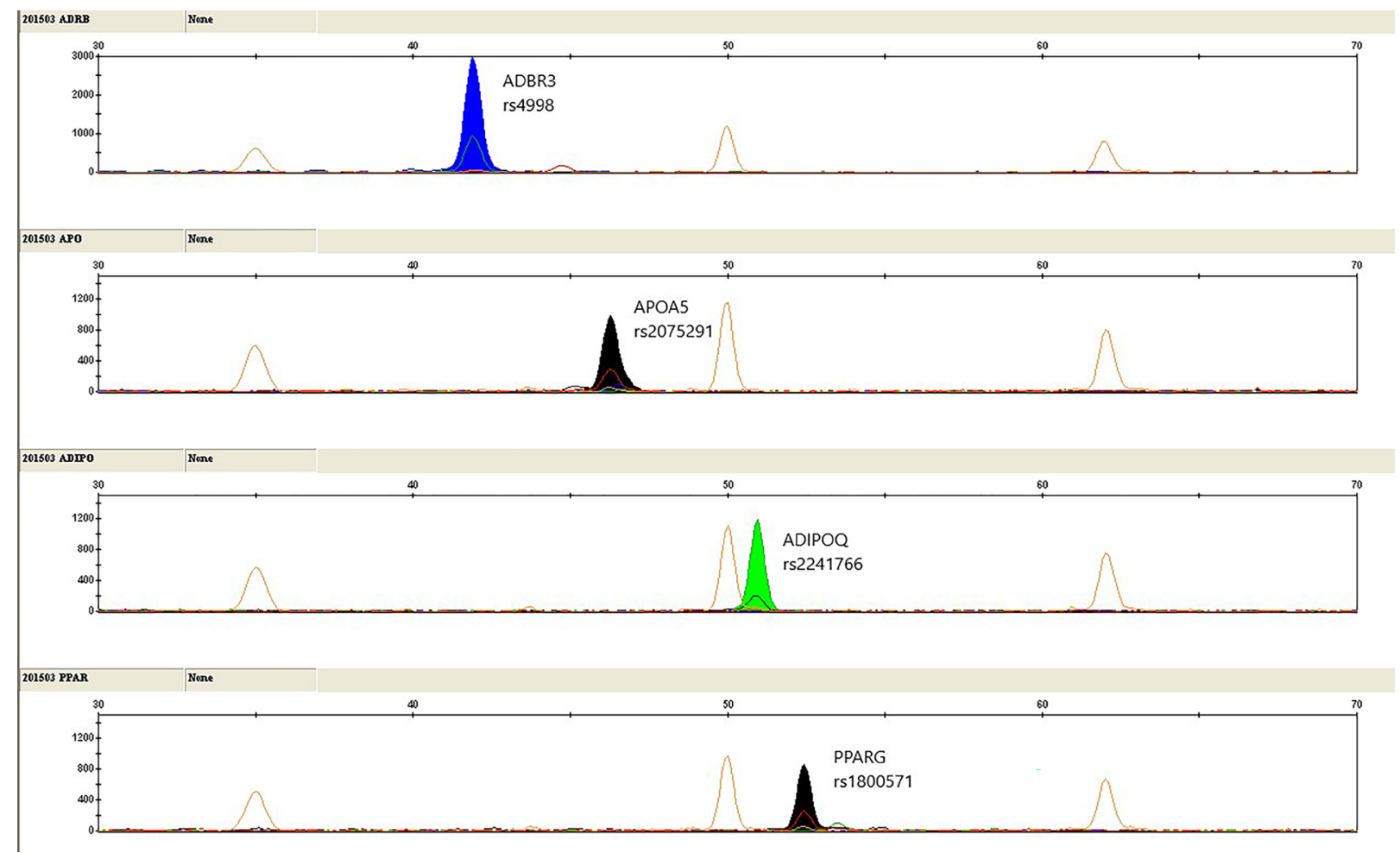

Figura 2. Identificación de cuatro polimorfismos amplificados en forma individual.

Algunos fragmentos mostraron una señal fluorescente más fuerte que otros debido a la química del fluorocromo con el que estaba marcado el ddNTP, sin embargo, este hecho no afectó la lectura del electroferograma. El análisis de los electroferogramas realizado por el software GeneMapper ${ }^{\circledR}$ del analizador genético ABI
310 permitió establecer el tamaño en pares de bases y la altura en unidades RFU de los picos. En la Tabla 3 se pueden observar las características que permitieron la detección de cada alelo, mediante la interpretación de los electroferogramas.

Tabla 3. Detección de las muestras en la electroforesis capilar.

\begin{tabular}{|c|c|c|c|c|c|c|c|}
\hline $\begin{array}{c}\text { Polimorfismo } \\
\text { (SNP) }\end{array}$ & Gen & Alelo & $\begin{array}{c}\text { Señal } \\
\text { fluorescente }\end{array}$ & $\begin{array}{c}\text { Tamaño } \\
(p b)\end{array}$ & $\begin{array}{c}\begin{array}{c}\text { Desviación } \\
\text { estándar (pb) }\end{array} \\
\end{array}$ & Mutación & Base detectada \\
\hline \multirow{2}{*}{$r s 4994$} & \multirow{2}{*}{ ADBR3 } & $\mathrm{T}$ & & 20 & 0.02 & \multirow{2}{*}{$A-->G$} & \multirow{2}{*}{ complementaria } \\
\hline & & $\mathrm{C}$ & & 22 & 0.01 & & \\
\hline \multirow{2}{*}{$r s 3135506$} & \multirow{2}{*}{ APOA5 } & G & & 26 & 0.07 & \multirow{2}{*}{ G-->C } & \multirow{2}{*}{ complementaria } \\
\hline & & $\mathrm{C}$ & & 27 & 0.06 & & \\
\hline \multirow{2}{*}{$r s 1501299$} & \multirow{2}{*}{ ADIPOQ } & $\mathrm{C}$ & & 33 & 0.08 & \multirow{2}{*}{ G-->T } & \multirow{2}{*}{ complementaria } \\
\hline & & A & & 34 & 0.09 & & \\
\hline \multirow{2}{*}{$r s 1801282$} & \multirow{2}{*}{ PPARG } & G & & 37 & 0.01 & \multirow{2}{*}{$\mathrm{C}-->\mathrm{G}$} & \multirow{2}{*}{ complementaria } \\
\hline & & $\mathrm{C}$ & & 37 & 0.08 & & \\
\hline \multirow{2}{*}{$r s 4998$} & \multirow{2}{*}{ ADBR3 } & G & & 41 & 0.08 & \multirow{2}{*}{$\mathrm{C}-->\mathrm{G}$} & \multirow{2}{*}{ complementaria } \\
\hline & & $\mathrm{C}$ & & 42 & 0.02 & & \\
\hline \multirow{2}{*}{$r s 2075291$} & \multirow{2}{*}{ APOA5 } & $\mathrm{C}$ & & 45 & 0.01 & \multirow{2}{*}{$\mathrm{C}-->\mathrm{A}$} & \multirow{2}{*}{ SNP } \\
\hline & & A & & 46 & 0.01 & & \\
\hline \multirow{2}{*}{$r s 2241766$} & \multirow{2}{*}{ ADIPOQ } & $\mathrm{C}$ & & 48 & 0.04 & \multirow{2}{*}{$\mathrm{T}-->\mathrm{G}$} & \multirow{2}{*}{ complementaria } \\
\hline & & A & & 50 & 0.07 & & \\
\hline \multirow{2}{*}{$r s 1800571$} & \multirow{2}{*}{ PPARG } & $\mathrm{C}$ & & 52 & 0.01 & \multirow{2}{*}{$\mathrm{C}-->\mathrm{A}$} & \multirow{2}{*}{ SNP } \\
\hline & & $\mathrm{A}$ & & 52 & 0.03 & & \\
\hline
\end{tabular}

Nota: el tamaño en $\mathrm{pb}$ corresponde al valor medio. 
En cada corrida se incluyeron controles negativos y positivos. En la Figura 3 se muestran los electroferogramas obtenidos de un control negativo, donde se observan únicamente los picos del marcador de peso molecular, y de un control positivo, donde se observan los picos de los alelos de cada polimorfismo detectado. El genotipo de cada polimorfismo se estableció mediante la asignación alélica realizada con base en el color del pico detectado, la altura del pico y al tamaño en pares de bases del fragmento. El método diseñado permitió detectar en cada uno de los polimorfismos, tanto del alelo ancestral como del alelo mutado, identificando así, perfiles homocigotos $\mathrm{y}$ heterocigotos en las muestras analizadas. En los electroferogramas obtenidos de tres de las muestras evaluadas, se muestran en la Figura 4 y en la Tabla 4 se observa el genotipo asignado a cada una de ellas.

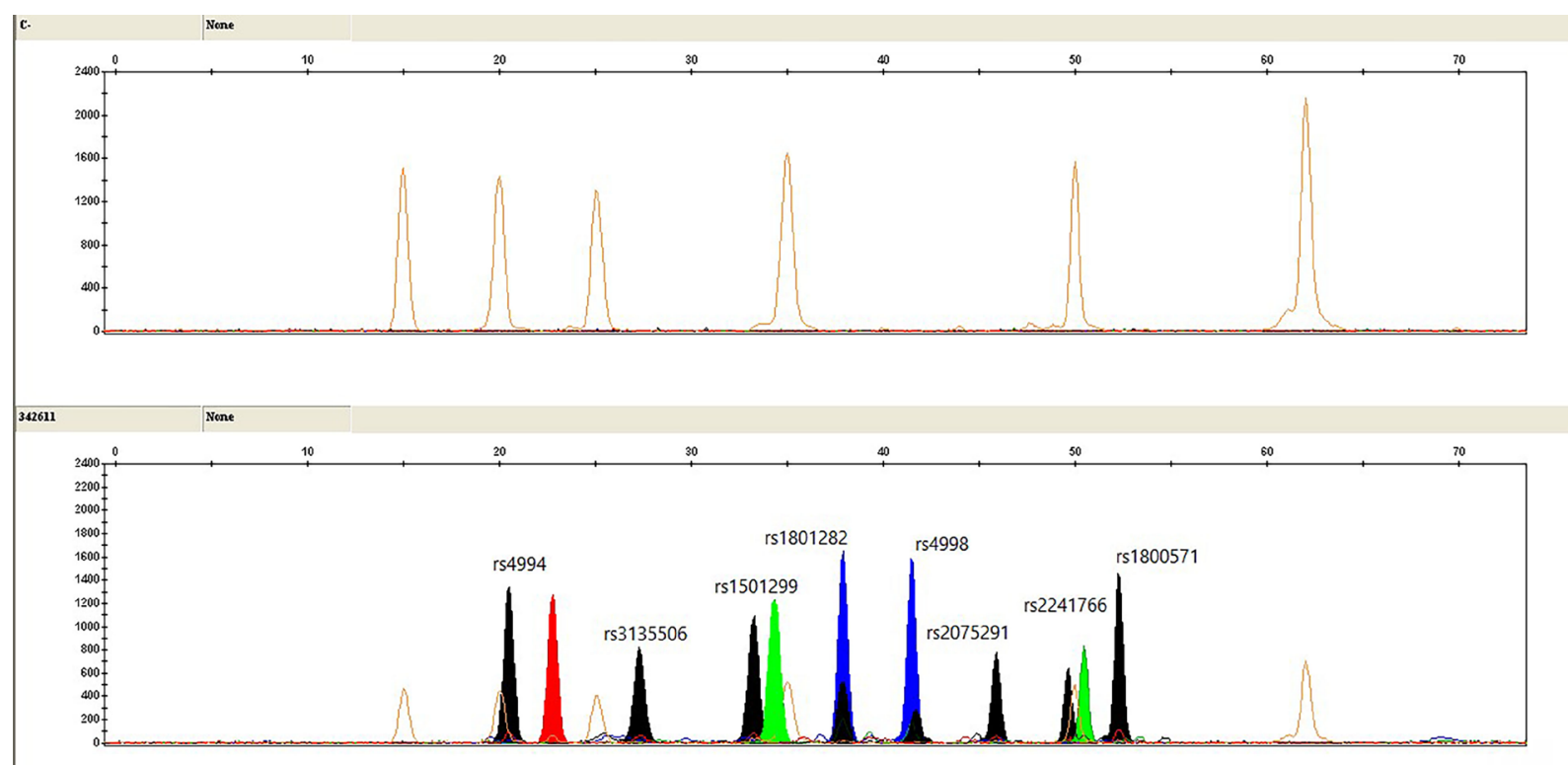

Figura 3: Arriba: Electroferograma de un control negativo con el marcador de peso molecular GeneScan ${ }^{\mathrm{TM}} 120$ LIZ ${ }^{\mathrm{TM}}$. Abajo: Electroferograma de un control positivo donde se visualiza el perfil genético de los polimorfismos de los genes ADBR3 ( $r s 4994$; rs4998), APOA5 (rs3135506; rs2075291), ADIPOQ (rs1501299, rs2241766) y PPARG (rs1801282, rs 1800571).

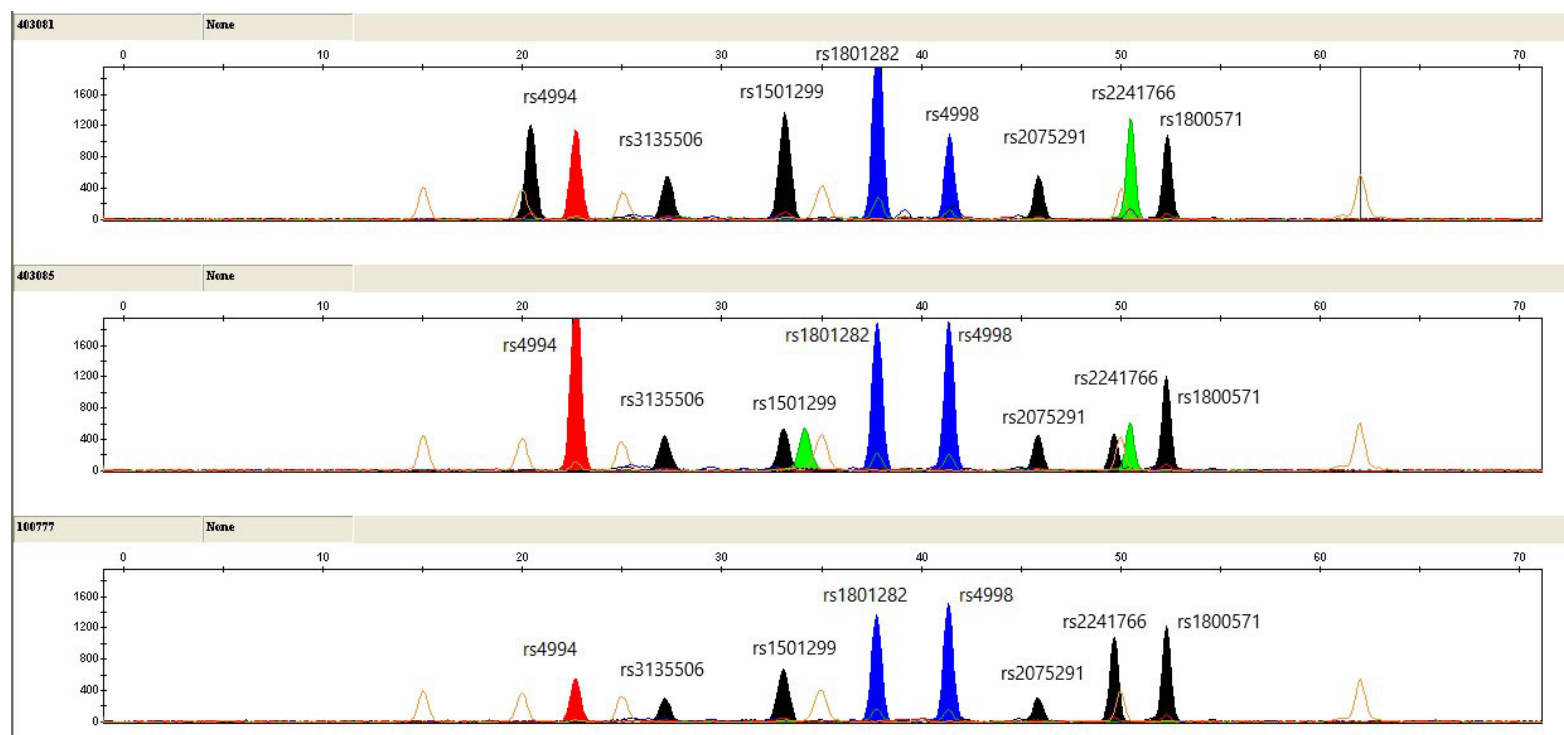

Figura 4. Electroferogramas obtenidos de tres muestras analizadas. Se visualiza el perfil genético de los polimorfismos de los genes ADBR3 (rs4994; rs4998), APOA5 (rs3135506; rs2075291), ADIPOQ (rs1501299, rs2241766) y PPARG (rs1801282; rs1800571). 
Tabla 4. Genotipos asignados a las muestras de la figura 4.

\begin{tabular}{ccccccccc}
\hline \multirow{2}{*}{ Muestra } & \multicolumn{7}{c}{ Genotipo } \\
\cline { 2 - 9 } & $\boldsymbol{r s 4 9 9 4}$ & $\boldsymbol{r} \mathbf{s 3 1 3 5 5 0 6}$ & $\boldsymbol{r s 1 5 0 1 2 9 9}$ & $\boldsymbol{r s 1 8 0 1 2 8 2}$ & $\boldsymbol{r s 4 9 9 8}$ & $\boldsymbol{r s 2 0 7 5 2 9 1}$ & $\boldsymbol{r s 2 2 4 1 7 6 6}$ & $\boldsymbol{r s 1 8 0 0 5 7 1}$ \\
\hline $\mathbf{4 0 3 0 8 1}$ & $\mathrm{A} / \mathrm{G}$ & $\mathrm{G} / \mathrm{G}$ & $\mathrm{G} / \mathrm{G}$ & $\mathrm{C} / \mathrm{C}$ & $\mathrm{C} / \mathrm{C}$ & $\mathrm{C} / \mathrm{C}$ & $\mathrm{T} / \mathrm{T}$ & $\mathrm{C} / \mathrm{C}$ \\
$\mathbf{4 0 3 0 8 5}$ & $\mathrm{A} / \mathrm{A}$ & $\mathrm{G} / \mathrm{G}$ & $\mathrm{G} / \mathrm{T}$ & $\mathrm{C} / \mathrm{C}$ & $\mathrm{C} / \mathrm{C}$ & $\mathrm{C} / \mathrm{C}$ & $\mathrm{T} / \mathrm{G}$ & $\mathrm{C} / \mathrm{C}$ \\
$\mathbf{1 0 0 7 7 7}$ & $\mathrm{A} / \mathrm{A}$ & $\mathrm{G} / \mathrm{G}$ & $\mathrm{G} / \mathrm{G}$ & $\mathrm{C} / \mathrm{C}$ & $\mathrm{C} / \mathrm{C}$ & $\mathrm{C} / \mathrm{C}$ & $\mathrm{G} / \mathrm{G}$ & $\mathrm{C} / \mathrm{C}$ \\
\hline
\end{tabular}

\section{Validación del ensayo}

Se realizó evaluando 20 controles y comparando el alelo detectado por secuenciación directa con el método de Sanger, usando el kit BigDye Terminator v3.1 Cycle Sequencing y el ensayo diseñado para minisecuenciación. En todos los casos el alelo detectado fue el mismo.

Con los electroferogramas obtenidos se determinó la altura y el peso en pares de bases de cada alelo y se calculó el promedio y desviación estándar. Las desviaciones estándar obtenidas estuvieron entre 0,01 y 0,09 pares de bases para los ocho SNPs, lo que permitió realizar la asignación alélica de manera inequívoca. El tamaño detectado para los alelos de cada polimorfismo, fue ligeramente diferente al tamaño esperado, sin embargo, esto no afectó en ningún caso la asignación alélica porque en el diseñó se tuvo en cuenta que la minisecuenciación de cada polimorfismo, generara fragmentos que difirieran en tamaño en cuatro o más pares de bases, descartando así la posibilidad de obtener picos sobrepuestos de dos SNPs adyacentes. A partir de la evaluación de la altura de los picos detectados en todos los controles se estableció el umbral analítico para asignar los alelos, en 100 unidades RFU.

La especificidad de cada par de cebadores diseñados, así como la exactitud del método de PCR múltiple diseñado, seguido de minisecuenciación por $\mathrm{SNaPshot}^{\circledR}$ se confirmó comparando los resultados obtenidos por secuenciación directa y por minisecuenciación de los productos amplificados. En los 20 controles evaluados el resultado de la secuenciación mostró que se amplificó la región esperada en cada uno de los genes y el alelo detectado fue idéntico.

Los resultados obtenidos de los cinco ensayos de una misma muestra demostraron la repetibilidad del método porque se detectaron invariablemente los mismos alelos en cada muestra, en todas las repeticiones analizadas. Así mismo los resultados obtenidos de la reproducibilidad del ensayo, evaluando las mismas muestras montadas y procesadas a ciegas, por un segundo funcionario, usando un termociclador dife- rente, arrojaron en todos los casos, el mismo resultado para el perfil genético de los ocho SNPs de interés mostrando la alta precisión del método.

Los electroferogramas obtenidos de las corridas electroforéticas fueron leídos y analizados por tres funcionarios de manera independiente y los perfiles asignados en todas las lecturas realizadas, fueron idénticos.

\section{Discusión y Conclusión}

El SM es el nombre dado al conjunto de condiciones clínicas que comprende obesidad central abdominal, hipertensión sistémica, insulina resistencia o diabetes mellitus tipo 2 y dislipidemia aterogénica. Es un estado protrombótico y proinflamatorio, asociado con aceleración de la enfermedad cardiovascular aterosclerótica, hiperuricemia o gota, enfermedad renal crónica y apnea obstructiva del sueño ${ }^{5}$. Es considerado de herencia multifactorial y se han reportado numerosos genes candidatos a estar asociados con algunos de los criterios para su diagnóstico; entre estos están los genes ADBR 3, APOA5, ADIPOQ y PPARG ${ }^{8,9,19}$.

En la literatura se han reportado estudios que asocian estos genes con el SM, sin embargo, ninguno de ellos evalúa en forma conjunta los cuatro genes, ni hay reportados estudios de asociación genética del SM en población latinoamericana, por lo que es indispensable realizar este tipo de investigaciones en nuestra población. La evaluación de muestras en estudios de asociación requiere de la tipificación de gran cantidad de muestras para lograr detectar la asociación y para ello se requiere contar con técnicas de genotipado que sean altamente específicas y que se permitan evaluar simultáneamente y de forma rápida, el mayor número posible de SNPs de interés, en un solo montaje ${ }^{25}$.

En este trabajo se presenta el diseño y validación de una PCR múltiple, seguida de minisecuenciación por SNaPshot, para detectar ocho polimorfismos ubicados en estos cuatro genes. El análisis de los SNPs mediante este ensayo diseñado y validado permitió 
la identificación de los alelos de cada polimorfismo usando esta metodología que es más sencilla, rápida y económica, que la secuenciación directa y los resultados fueron completamente reproducibles ${ }^{24}$.

Todos los cebadores se diseñaron para usarse específicamente en esta minisecuenciación múltiple, tuvieron un rendimiento óptimo y permitieron identificar en forma inequívoca cada uno de los alelos de los ocho SNPs. Así mismo la minisecuenciación por SNaPshot permitió corroborar que este método es altamente preciso dado el uso de ADN polimerasas para la incorporación de los nucleótidos y la posibilidad de desarrollar ensayos para la detección de varios polimorfismos ubicados en diferentes genes, en una sola reacción de PCR seguida de una sola minisecuenciación, lo cual optimiza tiempo y dinero ${ }^{2,25}$.

El ensayo también fue completamente validado siguiendo criterios de la norma ISO-NTC 17025:2017 y demostró ser altamente específico, exacto y reproducible, proporcionó resultados de identificación inequívoca de los SNPs sin ninguna identificación errónea ${ }^{2}$, lo que permite afirmar que el método de minisecuenciación con $\mathrm{SNaPshot}^{\circledR}$ descrito aquí, es un método simple pero robusto y preciso, que permite la detección rápida de múltiples polimorfismos y el análisis simultáneo de los ocho SNP. Ningún SNP experimentó un cambio de alelo en ninguna de las réplicas, y la posición en la que se detectaron los picos correspondientes a cada SNP, no sufrieron desplazamientos en los diferentes electroferogramas ${ }^{24,26,29,30}$.

La intensidad de las señales en la mayoría de los SNP presento variaciones, aunque fueron equilibradas, esto debido a la diferencia de intensidad de la señal emitida por el fluorocromo con que estaba marcado cada ddNTP ${ }^{25}$. También la movilidad de los fragmentos varió ligeramente las posiciones de los SNP en los electroferogramas, debido al tamaño de los fragmentos a detectar y al analizador genético, el polímero y tamaño del capilar, factores que afectan la movilidad electroforética ${ }^{25,28}$. Aunque estos aspectos en ningún momento dificultaron la asignación alélica de cada SNP, es importante realizar la validación del ensayo para establecer las condiciones de aceptabilidad, antes de ser usado para el análisis de muestras, adicionalmente consideramos indispensable, conservar todas las condiciones seguidas durante la validación, al analizar las muestras, para garantizar la reproducibilidad de los resultados. También es importante resaltar que para que un ensayo de detección múltiple de varios SNPs en una sola PCR seguida de una solo minisecuenciación sea robusto, se requiere optimizar el diseño y la concentración de los cebadores para la PCR y para la minisecuenciación de cada conjunto de SNP y, aunque esto demanda mucho tiempo y trabajo, permite la optimización del método ${ }^{2}$.

Finalmente consideramos que este trabajo aporta una herramienta de evaluación de estos polimorfismos con un diseño novedoso que podrá ser aplicado en un muestreo de población colombiana, para determinar si existe o no asociación de alguno de estos polimorfismos con el SM. Así mismo se constituye en un aporte para que otros investigadores desarrollen diseños mediante minisecuenciación para la detección de diversos SNPs de interés, dada la reproducibilidad, precisión, capacidad para analizar múltiples SNPs en forma simultánea, el consumo de tiempo y el costo por genotipo.

\section{Agradecimientos}

A las Doctoras Clara Inés Vargas, Adriana Gil y Adriana Pico por su colaboración para el desarrollo de este proyecto en las instalaciones del Laboratorio de Genética de la UIS y a la Doctora Leonor Gusmão por sus aportes en el diseño del estudio.

\section{Consideraciones éticas}

Este protocolo de investigación fue aprobado por el Comité de Ética de la Universidad Industrial de Santander (CEINCI). Todos los procedimientos siguieron los principios establecidos en la Declaración de Helsinki, de octubre de 2013, de la Asociación Médica Mundial, el Reporte Belmont, las pautas Éticas Internacionales para la Investigación Biomédica en Seres Humanos preparadas por el Consejo de Organizaciones Internacionales de Ciencias Médicas (CIOMS) en colaboración con la Organización Mundial de la Salud (OMS) del 2002, las Guías de Buenas Prácticas Clínicas de la Conferencia Internacional de Armonización. Además, estuvo regido por las normas científicas, técnicas y administrativas para la investigación en salud de la Resolución 008430 del 04 de octubre de 1993 del Ministerio de Salud y Protección Social de Colombia.

\section{Conflicto de intereses}

Los autores manifiestan no tener ningún conflicto de interés en el presente estudio. 


\section{Referencias}

1. Nachman MW, Crowell SL. Estimate of the mutation rate per nucleotide in humans. Genetics. 2000; 156(1): 297-304.

2. Sobrino B, Brion M, Carracedo A. SNPs in forensic genetics: a review on SNP typing methodologies. Forensic Sci Int. 2005; 154: 181-194. doi: 10.1016/j.forsciint.2004.10.020

3. Mehta B, Daniel R, Phillips C, McNevin D. Forensically relevant $\mathrm{SNaPshot}^{\mathbb{B}}$ assays for human DNA SNP analysis: a review. Int J Legal Med. 2017; 131(1): 21-37. doi: 10.1007/s00414-016-1490-5

4. Carlton V, Ireland J, Useche F, Faham M. Functional single nucleotide polymorphism-based association studies. Hum Genomics. 2006; 2(6): 391-402. doi: 10.1186/1479-7364-2-6-391

5. Samson S, Garber A. Metabolic Syndrome. Endocrinol Metab Clin N Am. 2014; 43: 1-23. doi: 10.1016/j.ecl.2013.09.009

6. Wakil S, Abu-Elheiga L. Fatty acid metabolism: target for metabolic syndrome. J Lipid Res. 2009; 50 Suppl(Suppl): S138-143. doi: 10.1194/j1r.R800079-JLR200

7. Goswami K, Gandhe M. Evolution of metabolic syndrome and its biomarkers. Diabetes Metab Syndr. 2018; 12(6): 1071-1074. doi: 10.1016/j.dsx.2018.06.027

8. Grygiel GB, Ziółkowska SI, Kaczmarek E, Mosor M, Nowak J, Puszczewicz M. PPARgamma-2 and ADRB3 polymorphisms in connective tissue diseases and lipid disorders. Clin Interv Aging. 2018; 13: 463-472. doi: 10.2147/CIA.S157186

9. Fiaz M, Shaiq P, Raj G, Saqlain M, Mehmood A, Muhammad S, et al. Association study of Apolipoprotein A5 gene (APOA5 gene) variant with the metabolic syndrome in local Pakistani population. J Pak Med Assoc. 2019; 69(3): 301-305.

10. Maen D, Abou Z, Arya M. Metabolic Syndrome: genetic insights into disease pathogenesis. Curr Opin Lipidol. 2016; 27(2): 162-171. doi: 10.1097/MOL.0000000000000276

11. Katsarou M, Karathanasopoulou A, Andrianopoulou A, Desiniotis V, Tzinis E, Dimitrakis E, et al. Beta 1, Beta 2 and Beta 3 Adrenergic receptor gene polymorphisms in a Southeastern European population. Front Genetics. 2018; 9: 560. doi: 10.3389/fgene.2018.00560

12. Vrablik M, Hubacek JA, Dlouha D, Satny M, Adamkova V, Ceska R. Strong Association between APOA5 Gene Polymorphisms and Hypertriglyceridaemic episodes. Folia Biol (Praha). 2019; 65(4): 188-194.
13. Aguilera CM, Gil-Campos M, Cañete R, Gil A. Alterations in plasma and tissue lipids associated with obesity and metabolic syndrome. Clin Sci. 2008; 114: 183-193. doi: 10.1042/CS20070115

14. Piya MK, McTernan PG, Kumar S. Adipokine inflammation and insulin resistance: the role of glucose, lipids and endotoxin. J Endocrinol. 2013; 216(1): T1-T15. doi: 10.1530/JOE-12-0498

15. Christodoulides C, Vidal-Puig A. PPARs and adipocyte function. Mole Cell Endocrinol. 2010; 318: 61-68. doi: 10.1016/j.mce.2009.09.014

16. Czech MP, Tencerova M, Pedersen DJ, Aouadi M. Insulin signalling mechanisms for triacylglycerol storage. Diabetologia. 2013; 56(5): 949-964. doi: 10.1007/s00125-013-2869-1

17. Schena G, Caplan MJ. Everything you always wanted to know about 3-AR. Cells. 2019; 8: 357. doi: 10.3390/cells 8040357

18. Huang M, Wang T, Lee K, Wu YJ, Tu HP, Liu CS, et al. Adiponectin gene snp276 variants and central obesity confer risks for hyperglycemia in indigenous Taiwanese. Kaohsiung J Med Sci. 2010; 26(5): 227236. doi: 10.1016/S1607-551X(10)70033-4

19. Brown AE, Walker M. Genetics of insulin resistance and the metabolic syndrome. Curr Cardiol Rep. 2016; 18(8): 75. doi: 10.1007/s11886-016-0755-4

20. Paneto G, Köhnemann S, Martins J, Cicarelli RM, Pfeiffer H. A single multiplex PCR and SNaPshot ${ }^{\circledR}$ minisequencing reaction of 42 SNPs to classify admixture populations into mitochondrial DNA haplogroups. Mitochondrion. 2011; 11(2): 296-302. doi: 10.1016/j.mito.2010.12.010

21. Koressaar T, Remm M. Enhancements and modifications of primer design program Primer3 Bioinformatics, 2007; 23(10): 1289-1291. doi: 10.1093/bioinformatics/btm091

22. Untergrasser A, Cutcutache I, Koressaar T, Ye $\mathrm{J}$, Faircloth B, Remm M, et al. Primer3 - new capabilities and interfaces. Nucleic Acids Res. 2012; 40(15): e115. doi: 10.1093/nar/gks596

23. Lindblad-Toh K, Winchester E, Daly MJ, Wang DG, Hirschhorn JN, Laviolette JP, et al. Largescale discovery and genotyping of single nucleotide polymorphisms in the mouse. Nat Genet. 2000; 24(4): 381-386. doi: 10.1038/74215

24. Lurkin I, Stoehr R, Hurst CD, van Tilborg A, Knowles MA, Hartmann A, et al. Two multiplex assays that simultaneously identify 22 possible mutation sites in the KRAS, BRAF, NRAS and PIK3CA genes. PLoS One. 2010; 5(1): e8802. doi: https://doi.org/10.1371/journal.pone.0008802 
25. Quintans B, Alvarez-Iglesias V, Salas A, Phillips C, Lareu MV, Carracedo A. Typing of mitochondrial DNA coding region SNPs of forensic and anthropological interest using $\mathrm{SNaPshot}$ minisequencing. Forensic Sci. Int. 2004; 14: 251257. doi: 10.1016/j.forsciint.2003.12.005

26. Børsting C, Rockenbauer E, Morling N. Validation of a single nucleotide polymorphism (SNP) typing assay with 49 SNPs for forensic genetic testing in a laboratory accredited according to the ISO 17025 standard. Forensic Sci Int Genetics. 2009; 4(1): 3442. doi: 10.1016/j.fsigen.2009.04.004

27. Bregu J, Conklin D, Coronado E, Terril M, Cotton R, Grgicak C, et al. Analytical thresholds and sensitivity: establishing RFU thresholds for forensic DNA analysis. J Forensic Sci. 2013; 58(1): 120-129. doi: 10.1111/1556-4029.12008

28. Palencia-Madrid L, Vinueza-Espinosa D, Baeta M, Roncandio A, Pancorbo MM. Validation of a 52-mtSNP minisequencing panel for haplogroup classification of forensic DNA samples. Int J Legal Med. 2020; 134(3): 929-936. doi: https://doi.org/10.1007/s00414-020-02264-6

29. Valverde-Villegas JM, De Medeiros RM, Almeida SE. Immunogenetic profiling of 23 SNPs of cytokine and chemokine receptor genes through a minisequencing technique: Design, development and validation. Int J Immunogenet. 2017; 44(3): 135-144. doi: 10.1111/iji.12314

30. Sanchez J, Børsting C, Hallenberg C, Buchard A, Hernández A, Morling N. Multiplex PCR and minisequencing of SNPs - a model with $35 \mathrm{Y}$ chromosome SNPs. Forensic Sci. Int. 2003; 137(1): 74-84. doi: 10.1016/S0379-0738(03)00299-8

\section{Abreviaturas y unidades de medida}

ADBR3: Receptor Beta 3-Adrenérgico.

ADIPOQ: Adiponectina.

ADN: $\quad$ Acido Desoxirribonucleico.

APOA5: Apolipoproteína A5.

ASO: Hibridización alélica específica.

CEINCI: Comité de Ética de la Universidad Industrial de Santander.

ddNTP: Dideoxirribonucleótido.

IUPAC: Unión Internacional de Química Pura y Aplicada.

MALDI-TOF: Espectrometría de masas.

MPS: $\quad$ Secuenciación masiva en paralelo.

OMS: $\quad$ Organización Mundial de la Salud.

PCR: Reacción en cadena de la polimerasa.

PPARG: Receptor activador de la proliferación de los peroxisomas tipo gamma.

RFU: Unidades de fluorescencia relativa.

SM: $\quad$ Síndrome metabólico.

SNP: $\quad$ Polimorfismos de nucleótido sencillo. 